\title{
Potentielles ressources alimentaires d'origine végétale des chimpanzés (Pan troglodytes verus) dans la zone forestière du Parc National du Mont Sangbé, Ouest de la cote d'ivoire
}

\author{
Kouamé Antoine N'GUESSAN*, Moné Jean Roméo Gauthier KALOU BI, \\ Yao Célestin KOUAKOU et Béné Jean-Claude KOFFI
}
Laboratoire de Biodiversité et Ecologie Tropicale, Unité de Formation et Recherches en Environnement, Université Jean Lorougnon Guédé, Daloa, BP 150 Daloa, Côte-d'Ivoire.
*Auteur correspondant ; E-mail : nguekoi@yahoo.fr, Tel : +2250708560960

Received: 02-05-2021 $\quad$ Accepted: 05-10-2021 $\quad$ Published: 30-10-2021

\section{RESUME}

La connaissance des ressources alimentaires consommées par une communauté animale dans son milieu de vie contribue énormément à la compréhension de l'éthologie et à la prise de décision de conservation de cette communauté. La présente étude qui s'est déroulée dans la zone forestière du Parc National du Mont Sangbé (PNMS) Ouest de la Côte d'Ivoire avait pour objectif de fournir les premières informations sur le régime alimentaire des chimpanzés de ce parc, par la détermination de la liste des potentielles ressources alimentaires d'origine végétale. La méthodologie a consisté à faire une recherche bibliographique, à mener des enquêtes auprès des populations riveraines et à faire des prospections pédestres dans le parc. A l'issue des différentes investigations, 17 espèces de plantes ont été répertoriées comme étant susceptibles d'être consommées par les chimpanzés du PNMS. Les principaux organes des plantes consommées sont : les fruits (59\%), les feuilles (23\%), les moelles de tige (18\%). Ces plantes ont été répertoriées au centre et au sud-est de la zone forestière du PNMS. Cette distribution de plantes coïncide avec celle des indices de présence des chimpanzés récoltés sur le terrain. Bien que ces informations préliminaires soient très utiles, il faut cependant noter qu'elles ne représentent que les potentielles ressources alimentaires des chimpanzés. Il serait donc souhaitable de conduire des travaux pouvant permettre d'observer les individus sur le terrain afin de connaître réellement le régime alimentaire des chimpanzés du PNMS.

(C) 2021 International Formulae Group. All rights reserved.

Mots clés : Primates, régime alimentaire, disponibilté alimentaire, domaine vital.

\begin{abstract}
Knowledge of the food resources consumed by an animal community in its living environment contributes enormously to the understanding of the ethology and to the conservation decision-making of this community. This study which took place in the forest zone of the Mont Sangbé National Park (PNMS) West of Côte d'Ivoire, aimed at providing the first information on the diet of chimpanzees in this park, by determining the list of potential food resources of plant origin. The methodology consisted in carrying out a bibliographical research, in carrying out surveys among neighboring populations and in carrying out pedestrian surveys in the park. At the end of the investigations, 17 species of plants were listed as being likely to be consumed by the
\end{abstract}


chimpanzees of the PNMS. The main organs of the plants consumed are: fruits (59\%), leaves (23\%), stem pith $(18 \%)$. These plants have been recorded in the center and south-east of the PNMS forest zone. This distribution of plants coincides with that of the presence indices of chimpanzees collected in the field. Although this preliminary information is very useful, it should be noted, however, that it only represents the potential food resources of chimpanzees. It would therefore be desirable to carry out work that could make it possible to observe individuals in the field in order to really know the diet of the chimpanzees in the PNMS.

(C) 2021 International Formulae Group. All rights reserved.

Keywords: Primates, diet, food availability, home range.

\section{INTRODUCTION}

Les grands singes anthropomorphes tiennent une place importante dans la compréhension de l'évolution des comportements humains du fait de leur proximité morphologique, comportementale et génétique avec l'homme (Edwards \& Beerli, 2000 ; Henry, 2019). Malheureusement, à l'instar des autres primates, ils sont aujourd'hui, en majorité, menacés d'extinction. Les Orang-Outan en Asie, les chimpanzés et les gorilles en Afrique, sont sous les énormes pressions de l'agriculture, du braconnage et des trafics divers (Humle et al., 2016). En Côte d'Ivoire, le chimpanzé, seule espèce de grands singes présente, est classé «en danger critique » d'extinction du fait de la disparition de son habitat naturel. La population de chimpanzés qui était estimée entre 8000 et 12000 individus en 1990 a connu une chute fulgurante d'environ $90 \%$ (Anderson, 2010 ; Kuehl et al., 2017). Les populations restantes sont confinées désormais dans des aires protégées qui subissent elles aussi les assauts dévastateurs des populations du fait de l'agriculture et du braconnage.

Ces menaces ont suscité des actions de conservation soutenues par des activités de recherches à différents niveaux. Cependant, en dehors des études d'estimation des effectifs des populations qui ont couvert l'ensemble du territoire ivoirien (Campbell et al., 2008), les travaux sur les comportements des chimpanzés se sont déroulés principalement au Parc National de Taï (PNT) au sud-ouest du pays, zone de forêt dense sempervirente. Ces études ont révélé des comportements spécifiques parmi lesquels certains ont des substrats écologiques et d'autres des origines culturelles (Lehmann \& Boesch, 2003). Il ressort aussi de ces études, que le choix alimentaire est largement influencé par la variation saisonnière de la disponibilité des ressources. Ainsi, quand leurs aliments préférés se font rares, ils adoptent différentes stratégies pour assurer leur survie (Zihlman et al., 2008). Dans certains cas, ils consomment de grandes quantités d'aliments de moins bonne qualité. Ce qui les oblige à augmenter le temps consacré à l'alimentation (Remis \& Kerr, 2002). Dans d'autres cas, ils peuvent soit augmenter la longueur de leurs déplacements quotidiens afin de trouver la ration du jour, avec une augmentation de la diversité de leur régime alimentaire, soit baisser les parcours quotidiens afin de minimiser les débauches d'énergie, avec une diminution de la diversité du régime alimentaire (Moore et al., 2018 ; Green et al., 2020).

Des initiatives dans le même sens, au stade préliminaire, sont en cours aujourd'hui dans le Parc National de la Comoé (PNC) au nord-est, en zone de savane (Lapuente et al., 2020) et quelques études se sont déroulées sur les chimpanzés de Yealé, au Mont Nimba (Humle, 2001).

Cependant aucune étude n'a été effectuée sur le comportement des chimpanzés du Parc National du Mont Sangbé (PNMS). Ce parc, situé à l'ouest du pays, fait partie des aires 
protégées ayant échappé au contrôle des gestionnaires durant la décennie de crise qu'a connu la Côte d'Ivoire. Cette situation a entrainé des dégâts environnementaux qui ont porté gravement atteinte à l'intégrité de ce parc (Normand \& Boesch, 2010). Pendant cette période, l'état de la population de chimpanzés et d'autres espèces fauniques était resté peu connu.

Pour éviter la disparition de certaines espèces emblématiques de ce parc dont les chimpanzés, des actions urgentes de conservation post-crises dont le programme de suivi écologique mis en œuvre depuis 2016 par la Direction de Zone ouest (DZO) de l'Office Ivoirien des Parcs et Réserves (OIPR) et des activités de recherches menées par l'Université Jean Lorougnon Guédé de Daloa (UJLoG), ont été initiées (Tiemoko, 2018). Ces différentes activées ont confirmé la présence de chimpanzés dans la partie forestière du PNMS (IOPR, 2019) et une étude portant sur la densité et l'aire de répartition du chimpanzé dans la partie forestière de ce parc a été réalisée (Koffi, 2020).

Pour assurer la conservation cette population de chimpanzés, il serait très utile de connaître son environnement, mais surtout les principales ressources alimentaires présentes sur le site. En effet, la quête de nourriture, moteur de toutes les autres activités, peut permettre de comprendre la vie dans la communauté et les relations avec les autres (Moscovice et al., 2007). Cette présente étude portant sur les chimpanzés de la zone forestière du PNMS, a pour objectif de fournir des informations sur leur régime alimentaire en vue de contribuer à leur sauvegarde au sein de ce parc.

\section{MATERIEL ET METHODES}

\section{Site d'étude}

Le Parc National du Mont Sangbé (PNMS) a une superficie totale de 95000 ha et est situé à l'Ouest de la Côte d'Ivoire entre $7^{\circ} 51^{\prime}$ ' et $8^{\circ} 10^{\prime}$ ' de latitude nord et $7^{\circ} 03^{\prime}$ ' et $7^{\circ} 23^{\prime}$ de longitude ouest. Il est à cheval entre les Sous-préfectures de Gbonné, Foungbesso, Guintéguéla et de Sifié (Figure 1). Le Parc National du Mont Sangbéa été classé le 19 février 1976. Ce parc se situe dans une région à deux saisons. Une saison sèche qui dure de cinq à six mois (Novembre à avril voire mai) accentuée par 2 à 3 mois d'harmattan et une saison pluvieuse qui s'étend sur cinq mois (Juin à octobre). Les précipitations annuelles varient entre 1100 et $1600 \mathrm{~mm}$. La température moyenne annuelle de la zone du parc est de $24,5^{\circ} \mathrm{C}$; ; L'humidité relative moyenne annuelle de la zone du parc est $75 \%$. C'est une zone montagneuse de transition savane forêt (Lauginie, 2007).

\section{Collecte des données}

Trois méthodes ont été utilisées pour la réalisation de ce travail en vue de maximiser la fiabilité des résultats. Ce sont: la recherche bibliographique, les enquêtes auprès des populations riveraines et la prospection pédestre. Il faut toutefois préciser que l'étude a été réalisée dans la zone forestière du PNMS, espace où les précédents travaux ont mentionné la présence de chimpanzés.

\section{Recherche bibliographique}

Des travaux de recherche sur le régime alimentaire des chimpanzés du Parc National de Taï (PNT) ont été consultés afin de sortir une liste de plantes régulièrement consommées par ceux-ci (N'guessan, 2012 ; Gone Bi et al., 2018). Les chimpanzés du PNT étant habitués à la présence humaine et suivis au quotidien, la liste des plantes qu'ils consomment régulièrement a servi de référence.

Ensuite, cette liste a été comparée à celle de la flore du PMNS, ce qui a permis d'obtenir une liste de plantes consommées par les chimpanzés à Taï et présentes au PNMS. 
Enquêtes auprès des populations riveraines

L'objectif de cette enquête a été d'obtenir auprès des populations le nom de certaines plantes connues pour être consommées par les chimpanzés et qui pourraient être absentes des listes bibliographiques. Pour cela, des villageois de deux villages (Kokialo et Tompoudé) situés à la périphérie de la zone forestière du PNMS ont été interrogés. Ces deux villages font partie des 12 villages ayant été délocalisés lors de la reprise en main du parc par les gestionnaires. Au cours de cette enquête, des personnes ayant séjourné dans le parc avant l'opération de délocalisation et des assistants de suivi écologique de l'OIPR (Guides du parc) ont été interrogés. Au total, 10 personnes ont été individuellement interrogées. Les enquêtes se sont déroulées avec l'accord préalable des chefs de village. Lorsqu' une personne à interroger est rencontrée, elle est tout d'abord rassurée en lui indiquant la raison de l'enquête afin d'établir une confiance. Une fois cela fait, les questions contenues dans le questionnaire lui sont posées. Les questions posées au cours de cette enquête s'articulaient autour des points suivants :

- L'existence de chimpanzés dans le parc ;

- Le nom des plantes locales consommées par les chimpanzés du parc.

\section{Prospection pédestre}

La prospection en forêt avait pour objectifs de confirmer la présence dans le parc des plantes signalées sur les différentes listes tout en marquant leurs coordonnées géographiques.

La prospection a été menée en utilisant la technique de marche de reconnaissance. Cette technique consiste à faire les observations en se déplaçant lentement et silencieusement à une vitesse d'environ 0,5 à 1 $\mathrm{km} / \mathrm{h}$, en empruntant des zones de moindre résistance (pistes d'animaux, pistes humaines, sous-bois clairs, lignes de crête, etc.). Chaque fois qu'un obstacle est rencontré, une déviation n'excédant pas $40^{\circ}$ peut être autorisée (White \& Tutin., 2001).

Lorsqu'une plante présente sur les listes est identifiée, le nom et les coordonnées géographiques sont marqués et une photographie est faite. Les coordonnées géographiques des indices de présences des chimpanzés rencontrés (restes alimentaires, empreintes, nids, vocalisations, crottes) sont aussi marquées et ces derniers sont photographiés.

\section{Analyse des données}

L'identification des plantes est basée sur les connaissances de la flore de la région et également à l'aide du guide illustré de la flore du PNT (Cellule d'Aménagement du PNT, 2000), de la liste de plantes régulièrement consommées par les chimpanzés du PNT (N'guessan, 2012 ; Gone Bi et al., 2018).

Les proportions et les graphes ont été réalisés grâce au logiciel EXEL.

La distribution spatiale des plantes consommées par les chimpanzés répertoriés sur le terrain a été réalisée grâce au logiciel QGIS2.14.3. 


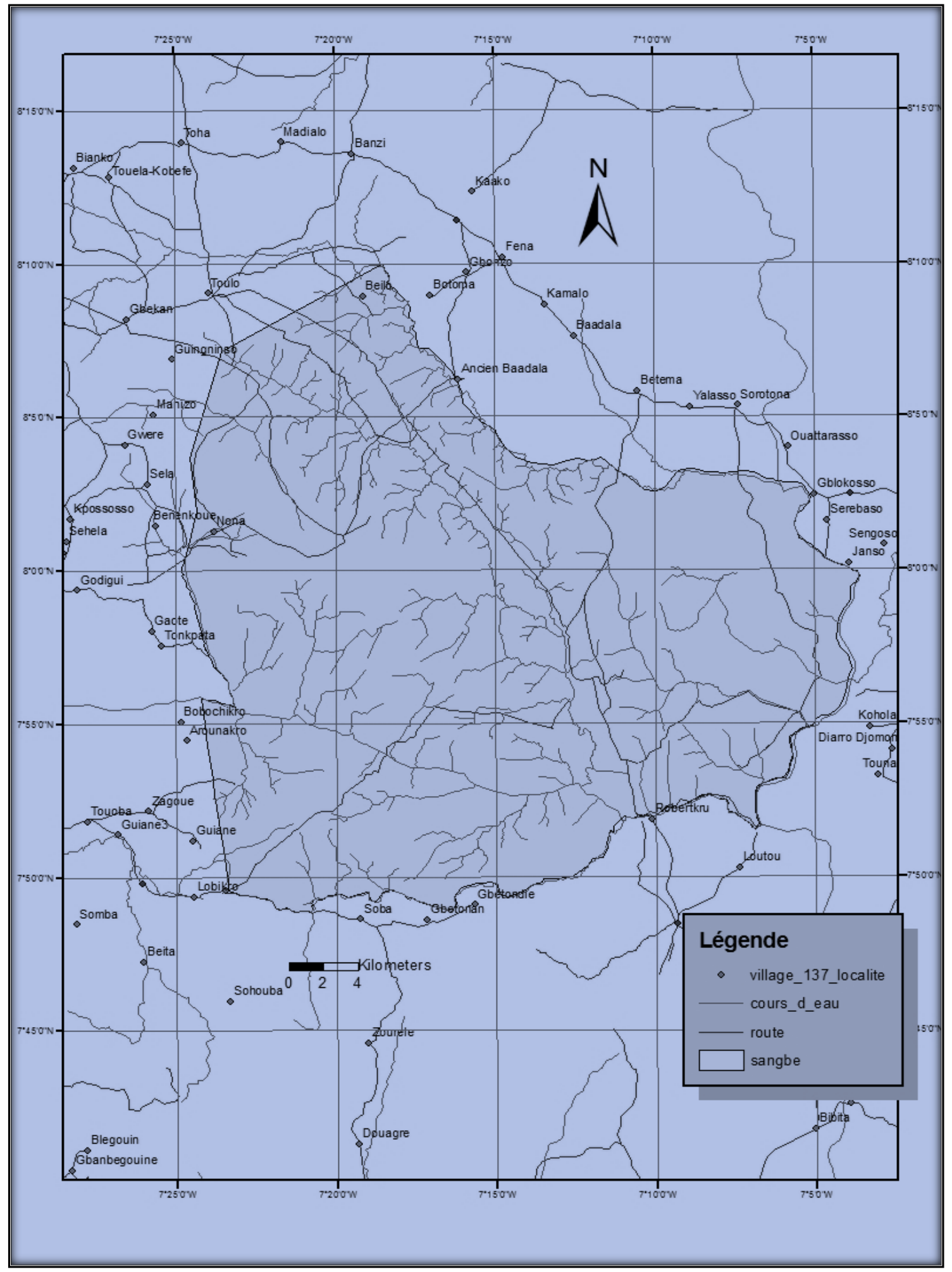

Figure 1 : Situation géographique du Parc National du Mont Sangbé.

Source : Tra Bi (2018). 


\section{RESULTATS}

\section{Potentielles ressources alimentaires} d'origine végétale des chimpanzés du PNMS

Les recherches bibliographiques ont permis de recenser 49 espèces de plantes appartenant à 26 familles consommées régulièrement par les chimpanzés du PNT, constituant ainsi la liste de référence. Les différentes parties consommées au niveau de ces plantes sont les fruits $(71,1 \%)$, les feuilles $(15,6 \%)$ et les moelles de tiges $(13,3 \%)$. Au niveau des fruits trois (3) espèces sont des noix cassées avec des outils et une espèce pour ces fruits et le noyau cassé plus tard avec des outils (Tableau 1).

Le croisement entre la liste de référence et la liste bibliographique de la flore du PNMS a permis d'identifier 16 espèces de plantes consommées par les chimpanzés de Taï et présentes au PNMS (Tableau 2). Il s'agit de plantes dont les parties consommées sont : les fruits, les feuilles et les moelles de tiges.

A l'issue des enquêtes, neuf (9) plantes ont été signalées comme consommées par les chimpanzés et susceptibles d'être rencontrées dans le PNMS. Il s'agit de plantes dont les organes consommés sont : les fruits (5), les feuilles (2) et les moelles de plantes (2). Une de ces neuf (9) plantes, Elaeis guineensis est consommée à la fois pour la moelle et les graines cassées avec des outils. De ces neuf (9) espèces seule une plante, Cola caricaefolia ne figure ni sur la liste de référence du PNT ni sur la liste bibliographique de la flore du PNMS.

La prospection pédestre a permis de confirmer sur le terrain la présence de 13 espèces de plantes dont les organes sont susceptibles d'être consommés par les chimpanzés du PNMS (Figure 2). Toutes ces 13 plantes sont présentes sur la liste bibliographique de la flore du PNMS.

En combinant les différents résultats, au total 17 espèces de plantes, appartenant à 11 familles, pouvant être considérées comme potentielles ressources alimentaires d'origine végétale des chimpanzés du PNMS ont été identifiées (Tableau 3).

Sur ces 17 plantes identifiées, 10 sont consommées au niveau de fruits (59\%), quatre (4) consommées au niveau des feuilles (23\%) et trois (3) consommées au niveau de la moelle (18\%) (Figure 3).

\section{Distribution Spatiale des ressources alimentaires d'origine végétale des chimpanzés du PNMS et des indices de présence de chimpanzés}

Au cours des prospections pédestres, les 13 espèces de plantes potentiellement consommées par les chimpanzés du PNMS ont été vues majoritairement dans la partie centrale de la zone forestière et aussi au sud-est de cette même zone (Figure 4).

Des indices de présence de chimpanzés ont été aussi rencontrés lors de la prospection pédestre (41 indices). Ces indices, tous indirectes, sont composés de restes alimentaires (15), de nids (21), d'empreintes (2) et de vocalisations (3). Les indices de présence ont été trouvés dans les parties centrale et sud-est de la zone forestière du PNMS.

Tableau 1: Plantes régulièrement consommées par les chimpanzés du PNT.

\begin{tabular}{llll}
\hline Nom Commun & \multicolumn{1}{c}{ Nom Scientifique } & \multicolumn{1}{c}{ Famille } & \multicolumn{1}{c}{ Partie consommée } \\
\hline Azodau & Afzelia bella & Caesalpiniaceae & $\mathrm{Fe}$ \\
\hline Rotin & Ancistrophyllum secondiflorum & Arecaceae & $\mathrm{m}$ \\
\hline Ako & Antiaris welwischii & Moraceae & $\mathrm{Fr}$ \\
\hline Duêh & Beilschmiedia mannii & Lauraceae & $\mathrm{Fr}$ \\
\hline Guépizou & Calpocalyx aubrevillei & Mimosaceae & $\mathrm{Fr}$ \\
\hline Guépizou & Calpocalyx brevibracteatus & Mimosaceae & $\mathrm{Fr}$ \\
\hline
\end{tabular}




\begin{tabular}{|c|c|c|c|}
\hline Anando & Chrysophyllum taiense & Sapotaceae & $\mathrm{Fr}$ \\
\hline Attia & Coula edulis & Olacaceae & Fr (Noix) \\
\hline Adjouaouba, & Dacryodes klaineana & Burseracea & $\mathrm{Fr}$ \\
\hline Bodo & Detarium senegalense & Caesalpiniaceae & Fr (Noix) \\
\hline Kropio & Dialium aubrevillei & Caesalpiniaceae & $\mathrm{Fr}$ \\
\hline Brobro & Dichapetalum pallidum & Chailletiaceae & $\mathrm{Fe}$ \\
\hline Igname sauvage & Dioscorea multiflora & Dioscoreaceae & $\mathrm{Fe}$ \\
\hline Otoumon & Duboscia viridiflora & Tiliaceae & $\mathrm{Fr}$ \\
\hline Palmier & Elaeis guineensis & Arecaceae & $\mathrm{m}$ \\
\hline Rotin & Eremospatha macrocarpa & Arecaceae & $\mathrm{m}$ \\
\hline figuier du Cap & Ficus $s p$ & Moraceae & $\mathrm{Fr}$ \\
\hline Kiokio & Glyphaea brevis & Tiliaceae & $\mathrm{Fe}$ \\
\hline Lopoum & Grewia bicolor & Tiliaceae & $\mathrm{Fr}$ \\
\hline Gloia & Halopegia azurea & Marantaceae & $\mathrm{m}$ \\
\hline Emballage & Hypselodelphys violacea & Marantaceae & $\mathrm{m}$ \\
\hline Boborou & Irvingia grandifolia & Irvingiaceae & $\mathrm{Fr}$ \\
\hline Kohaingué & Kaeyodendron brideloides & Euphorbiaceae & $\mathrm{Fr}$ \\
\hline Kroma & Klainedoxa gabonensis & Irvingiaceae & $\mathrm{Fr}$ \\
\hline Démouain & Landolphia hirsuta & Apocynaceae & $\mathrm{Fr}$ \\
\hline Démouain & Landolphia robustior & Apocynaceae & $\mathrm{Fe}$ \\
\hline Sidin & Magnistipula butayei & Chrysobalanaceae & $\mathrm{Fr}$ \\
\hline Vigo & Manniophython fulvum & Euphorbiaceae & $\mathrm{Fe}$ \\
\hline Trouyri & Memecylon sp & Melastomataceae & $\mathrm{Fr}$ \\
\hline Para solier & Musanga cecropiodes & Moraceae & $\mathrm{Fe}$ \\
\hline Badi & Nauclea diderrichii & Rubiaceae & $\mathrm{Fr}$ \\
\hline Badi & Nauclea xanthoxylon & Rubiaceae & Fr \\
\hline Aoukou & Panda oleosa & Pandaceae & Fr (Noix) \\
\hline Aramon & Parinari excelsa & Rosaceae & Fr (+ Noix) \\
\hline Néré de forêt & Parkia bicolor & Mimosaceae & $\mathrm{Fr}$ \\
\hline Ombre de forêt & Platysepalum hirsutum & Fabaceae & $\mathrm{Fr}$ \\
\hline Aningré & Pouteria aningeri & Sapotaceae & $\mathrm{Fr}$ \\
\hline Winyri & Pseudospondias microcarpa & Myristicaceae & $\mathrm{Fr}$ \\
\hline Ilomba, Oualélé & Pycnanthus angolensis & Myristicaceae & $\mathrm{Fr}$ \\
\hline Akouapo & Sacoglottis gabonensis & Humiriaceae & $\mathrm{Fr}$ \\
\hline Feuilles & Sarcophrynium sp & Marantaceae & $\mathrm{m}$ \\
\hline Akossika & Scotellia klaineana & Flacourtiaceae & $\mathrm{Fr}$ \\
\hline Lotofa & Sterculia oblonga & Sterculiaceae & $\mathrm{Fr}$ \\
\hline
\end{tabular}




\begin{tabular}{llll} 
Poé, koé & Strombosia pustulata & Olacaceae & $\mathrm{Fr}$ \\
\hline Blébléndou & Treculia africana & Moraceae & $\mathrm{Fr}$ \\
\hline Lowouhodin & Tricoscypha arborea & Anacardiaceae & $\mathrm{Fr}$ \\
\hline Banreléglénin & Tristemma coronatum & Melastomataceae & $\mathrm{Fr}$ \\
\hline Rikio, Borikio & Uapaca $s p$ & Euphorbiaceae & $\mathrm{Fr}$ \\
\hline Grattoir & Urera $s p$ & Urticaceae & $\mathrm{Fe}$ \\
\hline
\end{tabular}

Fr : Fruit ; Fe : Feuille ; $m$ : Moelle de plante

Tableau 2 : Plantes consommées par les chimpanzés du PNT et présentes au PNMS.

\begin{tabular}{llll}
\hline \multicolumn{1}{c}{ Nom commun } & \multicolumn{1}{c}{ Nom scientifique } & \multicolumn{1}{c}{ Famille } & Partie consommée \\
\hline Azodau & Afzelia bella & $\begin{array}{l}\text { Caesalpiniacea } \\
\text { e }\end{array}$ & $\mathrm{Fe}$ \\
\hline Palmier & Elaeis guineensis & Arecaceae & $\mathrm{m}$ \\
\hline Rotin & Eremospatha macrocarpa & Arecaceae & $\mathrm{m}$ \\
\hline Igname sauvage & Dioscorea multiflora & Dioscoreaceae & $\mathrm{Fe}$ \\
\hline Otoumon & Duboscia viridiflora & Tiliaceae & $\mathrm{Fr}$ \\
\hline figuier du Cap & Ficus sp & Moraceae & $\mathrm{Fr}$ \\
\hline Kiokio & Glyphaea brevis & Tiliaceae & $\mathrm{Fe}$ \\
\hline Démouain & Landolphia hirsuta & Apocynaceae & $\mathrm{Fr}$ \\
\hline Para solier & Musanga cecropiodes & Moraceae & $\mathrm{Fe}$ \\
\hline Badi & Nauclea xanthoxylon & Rubiaceae & $\mathrm{Fr}$ \\
\hline Lo, néré de forêt & Parkia bicolor & Mimosaceae & $\mathrm{Fr}$ \\
\hline Winyri & Pseudospondias microcarpa & Myristicaceae & $\mathrm{Fr}$ \\
\hline Ilomba, Oualélé & Pycnanthus angolensis & Myristicaceae & $\mathrm{Fr}$ \\
\hline Feuilles d'Attiéké & Sarcophrynium sp & Marantheceae & $\mathrm{m}$ \\
\hline Lotofa & Sterculia oblonga & Sterculiaceae & $\mathrm{Fr}$ \\
\hline Rikio, Borikio & Uapaca sp & Myristicaceae & $\mathrm{Fr}$ \\
\hline Fr : Fruit ; Fe: Feuille ; $:$ Moelle de plante & & \\
\hline & & & \\
\hline & & & \\
\hline
\end{tabular}



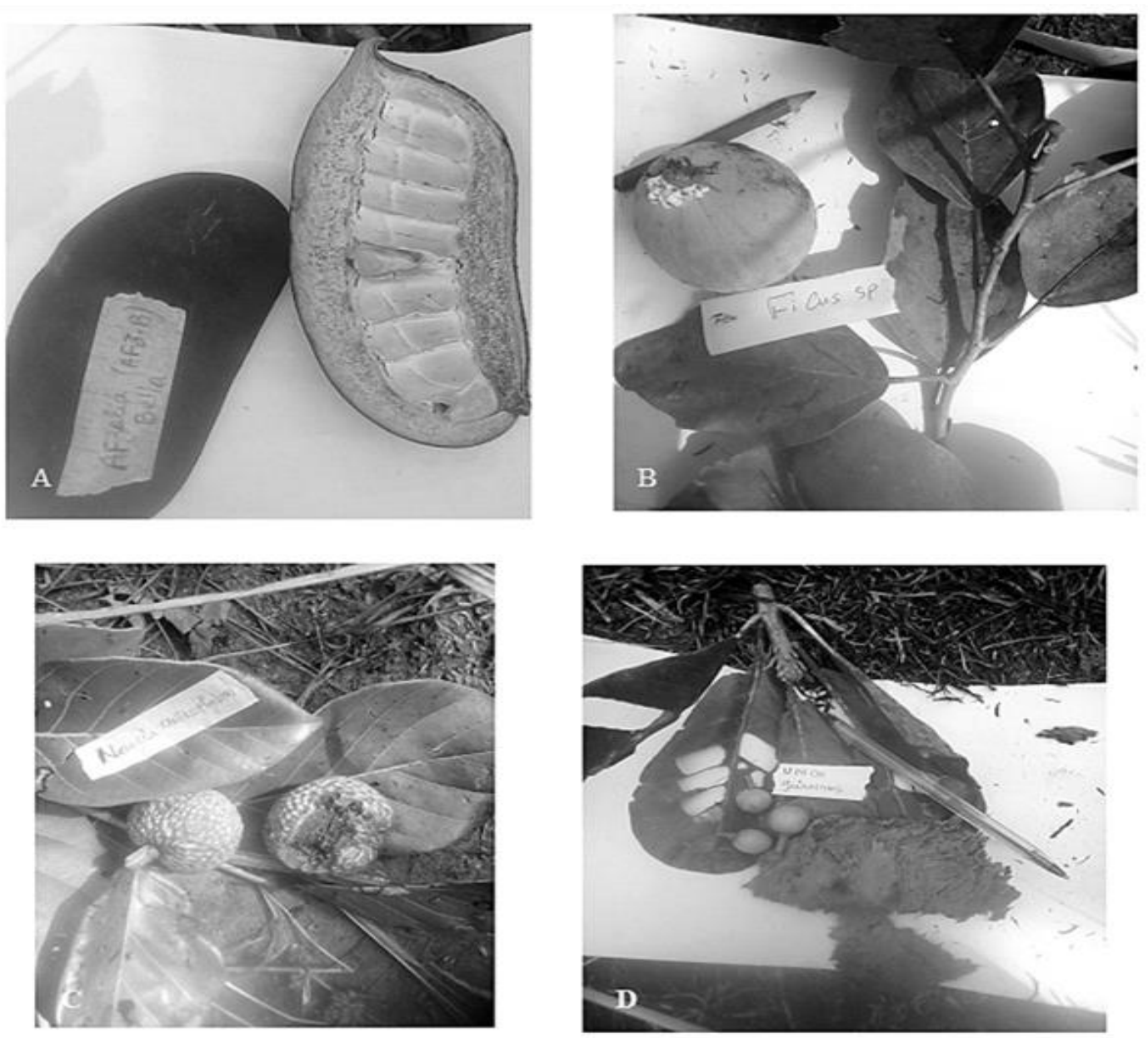

Figure 2 : Quelques organes de plantes potentiellement consommées par les chimpanzés du Parc National du Mont Sangbé (Kalou Bi).

A: Afzelia bella, B: Ficus sp, C: Nauclea xanthoxylon, D: Uapaca sp

Tableau 3: Plantes pouvant être considérées comme potentielles ressources alimentaires des chimpanzés du PNMS.

\begin{tabular}{llll}
\hline Nom commun & Nom scientifique & Famille & Partie consommée \\
\hline Azodau & Afzeliabella & Caesalpiniaceae & $\mathrm{Fe}$ \\
\hline Cola chimpanzé & Cola caricaefolia & Sterculiaceae & $\mathrm{Fr}$ \\
\hline Igname sauvage & Dioscorea multiflora & Dioscoreaceae & $\mathrm{Fe}$ \\
\hline Otoumon & Duboscia viridiflora & Tiliaceae & $\mathrm{Fr}$ \\
\hline Palmier & Elaeis guineensis & Arecaceae & $\mathrm{m}$ (+ noix) \\
\hline
\end{tabular}


K. A. N'GUESSAN et al. / Int. J. Biol. Chem. Sci. 15(5): 2058-2071, 2021

\begin{tabular}{llll}
\hline Rotin & Eremospatha macrocarpa & Arecaceae & $\mathrm{m}$ \\
\hline figuier du Cap & Ficus sp & Moraceae & $\mathrm{Fr}$ \\
\hline Kiokio & Glyphaea brevis & Tiliaceae & $\mathrm{Fe}$ \\
\hline Démouain & Landolphia hirsuta & Apocynaceae & $\mathrm{Fr}$ \\
\hline Parassolier & Musanga cecropiodes & Moraceae & $\mathrm{Fe}$ \\
\hline Badi & Nauclea xanthoxylon & Rubiaceae & $\mathrm{Fr}$ \\
\hline Néré de forêt & Parkia bicolor & Mimosaceae & $\mathrm{Fr}$ \\
\hline Winyri & Pseudospondias & Myristicaceae & $\mathrm{Fr}$ \\
\hline Ilomba & Pycnanthus angolensis & Myristicaceae & $\mathrm{Fr}$ \\
\hline Feuilles d'Attiéké & Sarcophrynium sp & Marantheceae & $\mathrm{m}$ \\
\hline Lotofa & Sterculia oblonga & Sterculiaceae & $\mathrm{Fr}$ \\
\hline Rikio, Borikio & Uapaca sp & Myristicaceae & $\mathrm{Fr}$ \\
\hline
\end{tabular}

Fr : Fruit ; Fe : Feuille ; $m$ : Moelle de plante

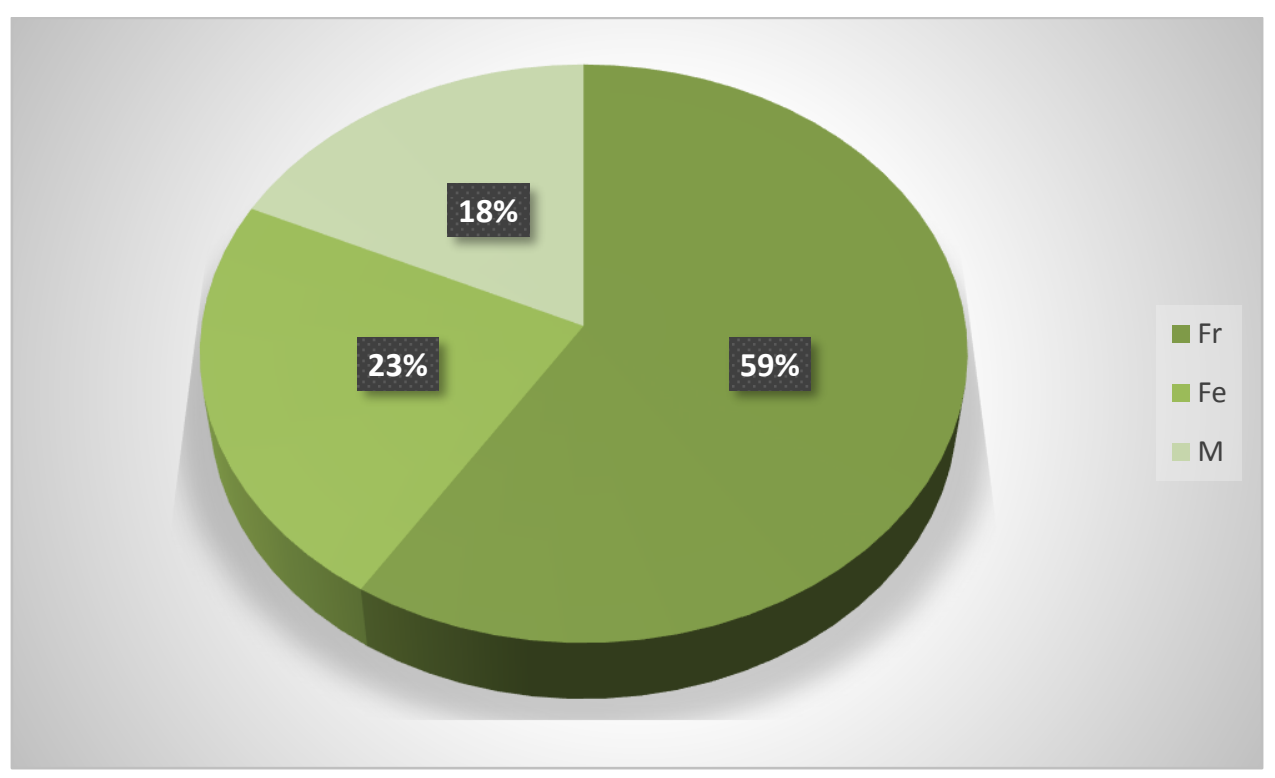

Figure 3 : Abondance relative des organes de plantes consommés par les chimpanzés du Parc National du Mont Sangbé.

Fr : Fruits, Fe : Feuilles, M : Moelles de plantes. 


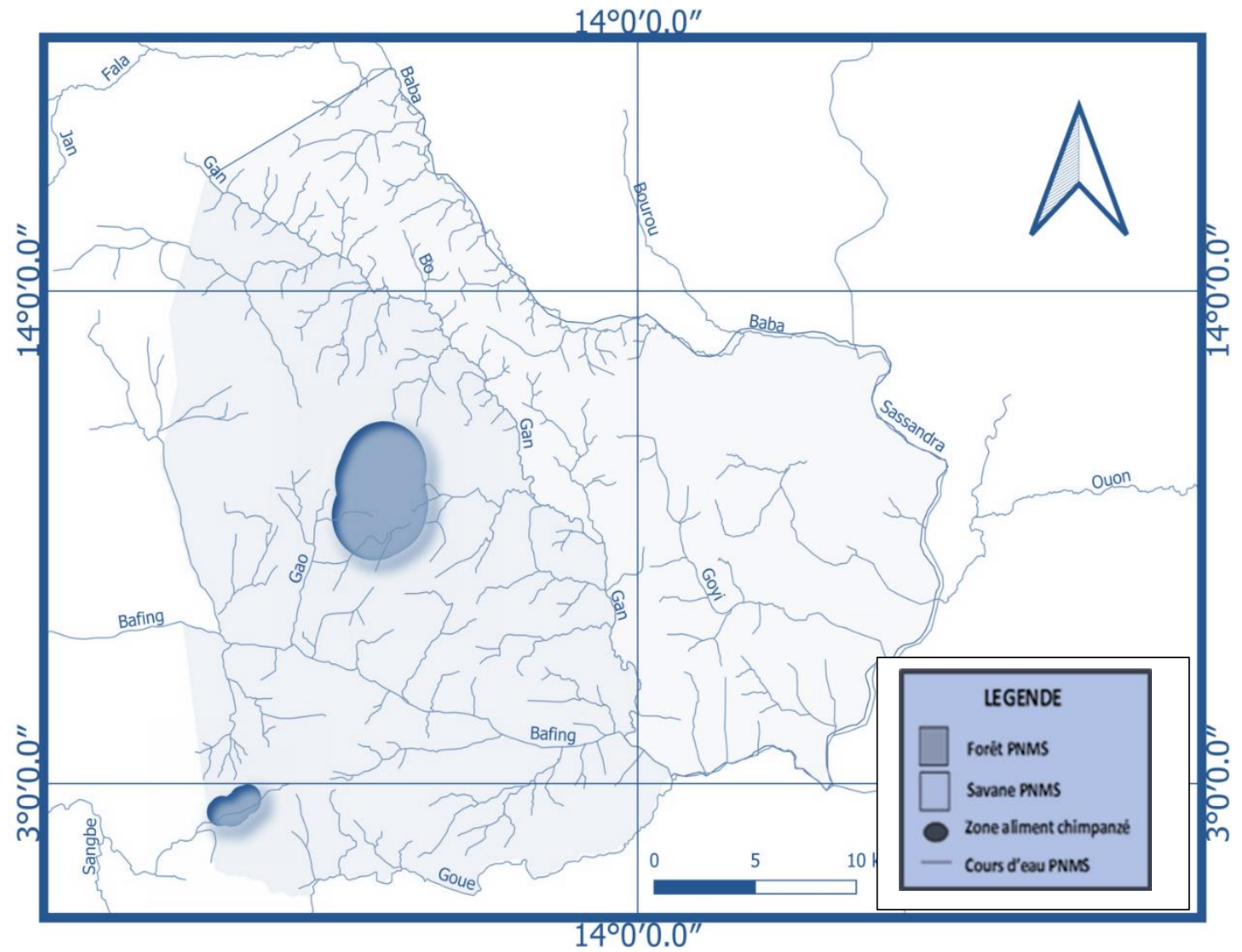

Figure 4 : Distribution spatiale des plantes consommées par les chimpanzés dans la zone forestière du Parc National du Mont Sangbé (Kalou Bi).

\section{DISCUSSION}

La combinaison des trois méthodes a permis d'identifier 17 espèces de plante pouvant constituer les potentielles ressources alimentaires d'origine végétale des chimpanzés du PNMS. Si 16 de ces plantes sont communes aux deux sites, une espèce (Cola caricaefolia) est censée être consommée uniquement par les chimpanzés du PNMS. En outre, les organes consommés au niveau de ces plantes sont les fruits (59\%), les feuilles (23\%) et des moelles de tiges $(18 \%)$. La valeur relativement faible du nombre d'espèce de plantes consommées par les chimpanzés au PNMS par rapport au PNT peut être due à la différence d'écosystème entre ces deux zones. En effet, le PNT est une forêt dense sempervirente alors que le PNMS est zone montagneuse de transition savaneforêt. Certaines plantes inféodées à la zone forestière peuvent ne pas proliférer dans cette zone. Aussi, les travaux réalisés sur l'alimentation des chimpanzés à Taï ont été faits durant de longues périodes sur des chimpanzés habitués à la présence humaine, permettant de faire des observations directes et détaillées (N'guessan, 2012 ; Gone Bi et al., 2018). La présente étude s'est déroulée sur une courte période en ne s'appuyant que sur des indices car les individus ne se laissent pas observer. Ce résultat est également loin des travaux réalisés par Hockings et al. (2009) à Bossou, en Guinée qui indiquent que 123 espèces de plantes sauvages constituent leur répertoire alimentaire des chimpanzés. Ce nombre élevé de Bossou ne fait toutefois pas de précision au niveau de la régularité de consommation de ces plantes comme c'est le cas au niveau du PNT. Il faut cependant noter 
que le nombre d'espèces de plantes consommées réellement par les chimpanzés du PNMS peut être supérieur à celui noté au cours de cette étude car la particularité écologique du site d'étude peut offrir des espèces uniquement consommées par les chimpanzés du PNMS comme c'est le cas de Cola caricaefolia, même si la consommation notée de cette plante reste à confirmer par une étude.

$\mathrm{La}$ nature des restes alimentaire (moelle, fruit) observés au cours de cette étude confirme la consommation des feuilles, des fruits et des moelles de plante par les chimpanzés du PNMS. Cette diversité d'organes de plantes entrant dans le régime alimentaire des chimpanzés du PNMS est similaire à plusieurs populations. En effet, un résumé de 24 études provenant de 11 sites donne les proportions suivantes pour les parties de plantes consommées : $64 \%$ de fruits, $16 \%$ de feuilles, $7 \%$ moelles de tiges, $3 \%$ de graines et $2 \%$ de fleurs (Conklin-Brittain et al., 2001). En plus, les enquêtes ont révélé que les chimpanzés du PNMS utilisent des outils pour la consommation des graines de palme. Ce comportement alimentaire particulier a été aussi vérifié chez les chimpanzés de Bossou et de Nimba (Biro et al., 2004). L'usage d'outil pour la consommation de certaines graines par les chimpanzés a été aussi observé à Taï (Luncz \& Boesch, 2014). L'utilisation d'outils pour casser les noix et les graines contribue énormément à leur alimentation et serait limitée aux populations de chimpanzés de l'Afrique de l'Ouest avec le fleuve Sassandra comme limite orientale (Proffitt et al., 2018). Cependant, les graines ou noix cassées diffèrent entre le PNT et le PNMS. Si à Taï, ce sont les noix de Coula edulis, Detarium senegalense, Panda oleosa et Parinari excelsa qui sont cassées (Boesch \& BoeschAchermann, 2000), au niveau du PNMS ce sont les graines de palme que les chimpanzés cassent avec des outils.

Les plantes constituant les potentielles ressources alimentaires des chimpanzés ont été répertoriées dans les zones centrale et sud-est de la zone forestière du PNMS. Les 41 indices de présence des chimpanzés ont été également enregistrés dans ces mêmes zones. Cette superposition de la distribution spatiale de ces deux paramètres indique que les chimpanzés sont bien présents dans la zone et utilisent ces plantes comme ressources alimentaires. Ces résultats sont conformes aux travaux menés récemment dans la même zone qui indiquent la présence de chimpanzés dans la zone forestière du PNMS (OIPR, 2019 ; Koffi, 2020). En plus de la présence des ressources alimentaires, cette présence dans ces zones pourrait être aussi justifiée par le refuge que constitueraient ces espaces loin des intrusions humaines (Bene et al., 2012). Le nombre relativement élevé d'indices sur un temps d'échantillonnage assez court fait penser à une densité de chimpanzé potentiellement élevée. Cette analyse est soutenue par des travaux antérieurs (Koffi, 2020) qui estiment la densité des populations de chimpanzés de cette zone à 0,48 Individu par $\mathrm{km}^{2}$, supérieure à celle du PNT qui est de 0,09 individu par km² (OIPR, 2019).

\section{Conclusion}

La recherche bibliographique, les enquêtes et la prospections pédestre employées au cours de cette étude ont permis d'établir une liste de plantes entrant dans le régime alimentaire des chimpanzés du PNMS et de déterminer leur distribution spatiale. Ainsi, 17 espèces de plantes ont été répertoriées comme étant susceptibles d'être consommées par les chimpanzés de ce parc. Les principaux organes de ces plantes consommées sont : les fruits $(56,2 \%)$, les feuilles (25\%), les moelles de tige $(18,8 \%)$. Des outils sont également utilisés pour casser des graines de palme. Ces plantes ont été répertoriées dans les parties centrale et sud-est de la zone forestière du PNMS. Cette distribution de plante coïncide avec celle des indices de présence des chimpanzés récoltés sur le terrain. Il faut cependant noter que ces informations représentent une ébauche dans la connaissance du régime alimentaire des chimpanzés du PNMS. Des travaux ultérieurs pouvant permettre de faire des observations directes doivent être envisagés. 


\section{CONFLIT D'INTERETS}

Les auteurs déclarent n'avoir aucun conflit d'intérêts sur cet article.

\section{CONTRIBUTIONS DES AUTEURS}

KAN et MJRGK ont conduit les travaux de terrain et ont effectué le traitement des données. YCK et BJCK ont participé à la conception de l'étude et ont supervisé le travail. Tous les auteurs ont participé à la rédaction et à la correction du manuscrit final.

\section{REMERCIEMENTS}

Les remerciements sont principalement adressés à l'Office Ivoirien des Parcs et Réserves (OIPR) pour avoir accepté la conduite de cette étude dans une de ses aires protégées et pour leur appui technique.

\section{REFERENCES}

Anderson J. 2010. Behavioral Pathologies in Nonhuman Primates. Encyclopedia of Behavioral Neuroscience, 72: 139-144. DOI: http://dx.doi.org/10.1016/B978-008-045396-5.00099-3

Bene JCK, Kone I, Gonedele Bi S, Bitty EA, Ouattara K, Akpatou KB, N'guessan KA, Koffi DA. 2012. The diurnal primate community of the Tanoé Forest: species composition, relative abundance, distribution, polyspecific associations and conservation status. Int. J. Biol. Chem. Sci., 6(1): 51-64. DOI: 10.4314/ijbcs.v6i1.5

Biro D, Inoue-Nakamura $\mathrm{N}$, Tonooka $\mathrm{R}$, Yamakoshi G, Sousa C, Matsuzawa T. 2003. Cultural innovation and transmission of tool use in wild chimpanzees: Evidence from field experiments. Animal Cognition, 6 (4): 213-223. DOI: 10.1007/s10071-0030183-x.

Boesch C, Boesch-Achermann H. 2000. The Chimpanzees of the Taï Forest: Behavioural Ecology and Evolution. Oxford University Press : Oxford, United Kingdom.

Campbell G, Kuehl H, N'goran KP, Boesch C. 2008. Alarming decline of West African chimpanzees in Côte d'Ivoire, Current Biology, 18: R903-R904. DOI: 10.1016/j.cub.2008.08.015 Cellule d'Aménagement du PNT: Flore du PNT. 2000. Edition: kasparek Verlag, p. 320.

Conklin-Brittain NL, Knott CD, Wrangham R. 2006. Energy intake by wild chimpanzees and orangutans: methodological considerations and preliminary comparison. In Feeding Ecology in Apes and other Primates, Hohmann G, Robbins M, Boesch C (Eds). Cambridge Univ. Press : Cambridge.

Edwards SV, Beerli P. 2000. Perspective : Gene divergence, population divergence, and the variance in coalescence time in phylogeographic studies. Evolution, 54: 1839-1854. DOI: $10.1111 /$ j.0014-3820. 2000.tb01231.x.

Gone Bi ZB, Vroh BTA, Adou Yao YC. 2018. Chimpanzee's (Pan troglodytes verus) activity and feeding patterns in Taï National Park, Cote d'Ivoire. SSRG International Journal of Agriculture \& Environmental Science, 5 (3) : 107-116. DOI: $\quad 10.14445 / 23942568 /$ IJAESV5I3P117.

Green SJ, Boruff BJ, Niyigaba P, Ndikubwimana I, Grueter CC. 2020. Chimpanzee ranging responses to fruit availability in a high-elevation environment. American Journal Primatology, 82(5): e23119. DOI: 10.1002/ajp.23119

Henry JP. 2019. Génétique et origine d'Homo sapiens. Medicine/science, 35: 39-45. DOI: $10.1051 /$ medsci/2018311

Hockings KJ, Anderson JR, Matsuzawa T. 2009. Use of wild and cultivated foods by chimpanzees at Bossou, republic of guinea: feeding dynamics in a humaninfluenced environment. American Journal of Primatology, 71: 636-646. DOI: 10.1002/ajp.20698

Humle T, Matsuzawa T. 2001. Behavioural diversity among the wild chimpanzee populations of Bossou and neighbouring areas, Guinea and Côte d'Ivoire, West 
Africa. Folia Primatologica, 27: 57-68. DOI: 10.1159/000049924

Humle T, Boesch C, Campbell G, Junker J, Koops K, Kuehl H, Sop T. 2016. Pan troglodytes verus. The IUCN Red List of Threatened species 2016.

Kuehl HS, Sop T, Williamson EA, Mundry R, Brugière D, Campbell G, Cohen H, Danquah E, Ginn L, Herbinger I, Jones S, Junker J, Kormos R, Kouakou CY, N'goran PK, Normand E, Shutt-phillips K, Tickle A, Vandras E, Welsh A, Wessling EG, Boesch C. 2017. The critically endangered western chimpanzee declines by $80 \%$. American Journal of Primatology, 79 (9): e22681. DOI: 10.1002/ajp.22681

Lapuente J, Ouattara A, Köster PC, Linsenmair KE. 2020. Status and distribution of Comoe Chimpanzees: combined use of transects and camera traps to quantify a low-density population in savanna-forest mosaic. Primates, 61 (4): 1-13. DOI: 10.1007/s10329-019-00786-1

Lauginie F. 2007. Conservation de la nature et Aires Protégées en Côte d'Ivoire. NEI/Hachette et Afrique Nature : Abidjan ; p. 668.

Lehmann J, Boesch C. 2003. Social influences on ranging patterns among chimpanzees (Pan troglodytes verus) in the Taï National Park, Côte d'lvoire. Behavioral Ecology, 14: 642-649. DOI : 10.1093/beheco/arg047

Luncz LV, Boesch C. 2014. Tradition over trend: Neighboring chimpanzee communities maintain differences in cultural behavior despite frequent immigration of adult females. American Journal of Primatology, 76 (7): 649-657. DOI: 10.1002/ajp.22259

Moore JF, Mulindahabi F, Gatorano G, Niyigaba P, Ndikubwimana I, Cipolletta C, Masozera MK. 2018. Shifting through the forest: home range, movement patterns, and diet of the eastern chimpanzee (Pan troglodytes schweinfurthii) in Nyungwe National
Park, Rwanda. American Journal of Primatology, 80(8): e22897. DOI: 10.1002/ajp.22897

Moscovice LR, Issa MH, Petrzelkova KJ, Keuler NS, Snowdon CT, Huffman MA. 2007. Fruit availability, chimpanzee diet, and grouping patterns on Rubondo Island, Tanzania. American Journal Primatology, 69(5): 487-502. DOI: 10.1002/ajp.20350

Normand E, Boesch C. 2010. Rapport de suivi écologique dans les forêts classées de Goin-Débé et de Cavally (2007-2010). Rapport d'activité WCF, p. 42.

N'Guessan KA. 2012. Aspectes qualitatifs et quantitatifs du régime alimentaire des chimpanzés (Pan troglodytes verus, Blumenbach 1779) au Parc National de Taï, Côte d'Ivoire. Thèse de Doctorat, UFR Biosciences, Université de Cocody, Abidjan, Côte d'Ivoire, p. 99.

OIPR. 2019. Présentation des données de la phase III de suivi écologique. Rapport d'activité, Parc National du Mont sangbé, Côte d'Ivoire, Man, p. 27.

Proffitt T, Haslam M, Mercader JF, Boesch C, Luncz LV. 2018. Revisiting Panda 100, the first archaeological chimpanzee nutcracking site. Journal of Human Evolution, 124: 117-139. DOI : 10.1016/J.JHEVOL.2018.04.016

Remis M, Kerr ME. 2002. Taste responses to fructose and tannic acid among gorillas (Gorilla gorilla gorilla). International Journal of Primatology, 23: 251- 261. DOI: 10.1023/A:1013827310497

Tiemoko DBC. 2018. Suivi de la population de l'hippotrague rouan (Hippotragus equinus, desmarest 1804) et d'autres mammiferes par piegeage photographique au Parc National du Mont Sangbe, à l'ouest de la Côte d'Ivoire. Mémoire de Master en Gestion et Conservation Durable de la Faune Sauvage, UFR Environnement, Université Jean Lorougnon Guédé, Daloa, Côte d'Ivoire, p. 60. 